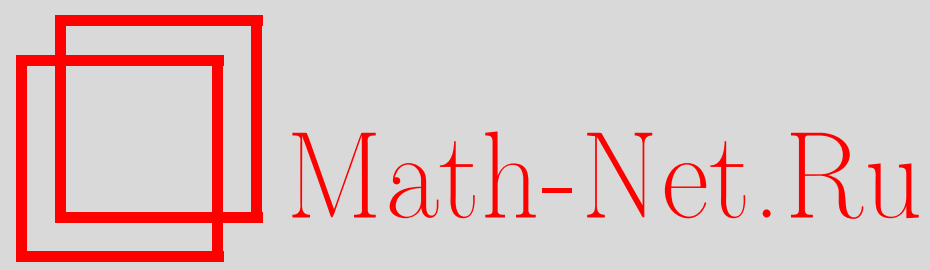

К. П. Харт, Элементарность и размерности, Матем. заметки, 2005, том 78, выпуск 2, 292-298

DOI: https://doi.org/10.4213/mzm2578

Использование Общероссийского математического портала Math-Net.Ru подразумевает, что вы прочитали и согласны с пользовательским соглашением http://www . mathnet.ru/rus/agreement

Параметры загрузки:

IP : 54.81 .137 .203

26 апреля 2023 г., 16:43:04

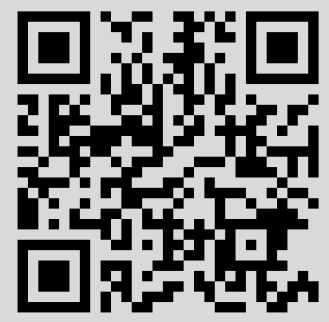


УДК 517.5

\title{
ЭЛЕМЕНТАРНОСТЬ И РАЗМЕРНОСТИ
}

\author{
K. П. Харт
}

Предложено альтернативное доказательство неравенства $\operatorname{dim} X \leqslant \operatorname{Dg} X$ для бикомпактов $X$, недавно полученного В.В. Федорчуком. Проблема сведена к метризуемому случаю при помощи теоремы Лёвенгейма-Скулема.

Библиография: 10 названий.

\section{1. Введение}

Среди существующих топологических определений размерности наибольшей известностью и популярностью пользуются Ind и ind (большая и малая индуктивные размерности), а также $\operatorname{dim}$ (лебегова размерность). Они отражают разные аспекты интуитивного понимания размерности. Индуктивные размерности формализуют представление о том, что “линию можно разбить точками, поверхность - линиями, апространствоповерхностями", тогда как $\operatorname{dim}$ - это формализация "числа возможных направлений"; это особенно хорошо видно из теоремы о перегородках $[1$, п. 7.2.15]. Все эти размерностные функции принимают одинаковые значения на всех метрических сепарабельных пространствах, причем размерность евклидова $n$-мерного пространства всегда оказывается "правильной".

В статье [2] Брауэр предложил другое понятие размерности, Dimensionsgrad (Dg), основанное на разрезах. Только недавно [3] было установлено, что Dg совпадает с общепринятьми размерностями на классе (локально) компактных метрических пространств. Вне этого класса Dg отличается от привычных размерностей; так, для каждого $n$ существует локально связное полное метрическое сепарабельное пространство $X_{n}$, для которого $\operatorname{Dg} X_{n}=1$ и $\operatorname{dim} X_{n}=n$ (см. [4]).

Недавно В. В. Федорчук доказал, что $\operatorname{dim} X \leqslant \operatorname{Dg} X$ для всех бикомпактов $X$. В этой статье мы передоказываем этот результат, а также неравенство Веденисова $\operatorname{dim} X \leqslant$ Ind $X$ (для нормальных пространств) средствами теории моделей.

Рассуждения, проведенные в этой статье, заставляют думать, что размерность Dg несколько сложнее общепринятых размерностей. Это позволяет объяснить, почему доказательство Федорчука настолько труднее относительно простого доказательства неравенства Веденисова.

\section{2. Предварительные сведения}

2.1. Размерности. Ниже мы напоминаем определения лебеговой размерности и большой индуктивной размерности. Говорят, что лебегов а размерность нормального 
пространства $X$ не превосходит $n(\operatorname{dim} X \leqslant n)$, если во всякое конечное открытое покрытие этого пространства можно вписать открытое покрытие кратности не более $n$ (т.е. такое, что никакая точка не принадлежит более чем $n+1$ элементам вписанного покрытия). Как обычно, размерность $\operatorname{dim} X$ определяется как наименьшее $n$, для которого $\operatorname{dim} X \leqslant n$ (если такого $n$ не существует, то мы полагаем $\operatorname{dim} X=\infty)$.

Большая индуктивная размерность определяется рекурсивно: Ind $X \leqslant n$ означает, что между любьми двумя непересекающимися замкнутьми множествами $A$ и $B$ есть перегородка $C$ размерности Ind $C \leqslant n-1 ; C$ назьвается перегородкой между $A$ и $B$, если $X \backslash C$ можно представить как объединение двух непересекаюшихся открытых множеств $U$ и $V$ таких, что $A \subseteq U$ и $B \subseteq V$. Основанием рекурсии служит неравенство Ind $X \leqslant-1$ для $X=\varnothing$.

Размерность Dimensionsgrad определяется подобньп образом, только теперь множество $C$ должно быть разрезом между $A$ и $B$; это означает, что оно замкнуто и пересекается с каждым континуумом, пересекающим оба множества $A$ и $B$.

2.2. Решетки. В статье [5] Волмэн показал, что каждой дистрибутивной решетке $L$ с $\mathbf{0}$ и 1 можно поставить в соответствие бикомпактное $T_{1}$-пространство $w L$, так назьваемое волмәновское представление этой решетки, замкнутой базой топологии которого является гомоморфньй образ решетки $L$. Множество $w L$ совпадает со множеством всех ультрафильтров на $L$; для каждого элемента $a$ решетки $L$ множество $\bar{a}=\{u \in w L: a \in u\}$ является элементом замкнутой базы топологии на $w L$. Гомоморфизм $a \mapsto \bar{a}$ взаимнооднозначен тогда и только тогда, когда решетка $L$ cenapaтивна, т.е. для любых $a \nless b$ найдется $c \leqslant a$ такое, что $c>0$ и $c \sqcap b=\mathbf{0}$. Пространство $w L$ хаусдорфово, если и только если $L$ нормальна, т.е. для любых $a \sqcap b=\mathbf{0}$ найдутся $f$ и $g$ такие, что $a \sqcap f=\mathbf{0}, b \sqcap g=\mathbf{0}$ и $f \sqcup g=\mathbf{1}$.

В отличие от стоуновского представления булевых алгебр, соответствие между решетками и их волмэновскими представлениями не взаимнооднозначно. Разумеется, каждое бикомпактное $T_{1}$-пространство $X$ является представлением своей собственной решетки замкнутых множеств (мы обозначаем ее через $2^{X}$ ); кроме того, $X=w \mathscr{B}$ для любой базы $\mathscr{B}$ замкнутых множеств в $X$, которая замкнута относительно конечных объединений и пересечений. Например, единичньй отрезок является волмэновским представлением семейства конечных объединений отрезков с рациональньми концами.

2.3. Элементарные подрешетки. Предлагаемые в этой статье доказательства неравенств Федорчука и Веденисова используют элементарные подрешетки решетки $2^{X}$. Подрешетка $L$ в $2^{X}$ назьвается әлементарной, если всякое разрешимое в $2^{X}$ уравнение с параметрами из $L$ разрешимо уже в $L$. Термин "уравнение" понимается в очень широком смысле. Имеется в виду вот что: если $\phi(x, y, \ldots, a, b, \ldots)$ - теоретико-решеточная формула со свободными переменными $x, y, \ldots$ и параметрами $a, b, \ldots$ из $L$ и если существуют $x, y, \ldots$ в $2^{X}$, при подстановке которых $\phi$ становится истинной формулой в $2^{X}$, то такие $x, y, \ldots$ найдутся и в $L$.

Всякая элементарная подрешетка в $2^{X}$ автоматически сепаративна: если $a \nless b$ в $L$, то

$$
(x \leqslant a) \wedge(x>\mathbf{0}) \wedge(x \sqcap b=\mathbf{0})
$$

является разрешимьм в $2^{X}$ уравнением с параметрами $(a, b$ и 0$)$ из $L$; значит, должно найтись $c \in L$, для которого

$$
(c \leqslant a) \wedge(c>0) \wedge(c \sqcap b=\mathbf{0}) .
$$


Точно так же, подрешетка $L$ должна быть нормальной: если $a, b \in L$ и $a \sqcap b=\mathbf{0}$, то уравнение

$$
(a \sqcap x) \wedge(b \sqcap y) \wedge(x \sqcup y=\mathbf{1})
$$

разрешимо в $2^{X}$; значит, сушествуют $f$ и $g$ из $L$, для которых

$$
(a \sqcap f) \wedge(b \sqcap g) \wedge(f \sqcup g=\mathbf{1}) .
$$

Ниже, при доказательстве неравенства Федорчука, мы встретимся с более сложньми уравнениями/формулами, содержашими кванторы; именно здесь проявится сила понятия элементарности.

Важную роль в теории моделей играет теорема Лёвенгейма-Скулема; в применении к решеткам она звучит так: для любого подсемейства $\mathscr{F}$ в $2^{X}$ найдется элементарная подрешетка $L$ решетки $2^{X}$ такая, что $\mathscr{F} \subseteq L$ и $|L| \leqslant|\mathscr{F}| \cdot \aleph_{0}$. Эта теорема дает возможность доказать факторизационную теорему Мардешича в сильной форме (примером ее применения служит [6, теорема 5.3]; систематическое исследование свойств, которые факторизующее пространство наследует из области определения факторизуемого отображения, проведено в диссертации [7]). Доказательство теоремы Лёвенгейма-Скулема можно найти в [8, п. 3.1].

\section{3. Формулы для размерностей}

3.1. Лебегова размерность. Мывоспользуемся принадлежащей Хеммингсену характеристикой [9] (см. также [1, следствие 7.2.14]), чтобы вьвести теоретико-решеточную формулу, характеризующую лебегову размерность в терминах замкнутых множеств. Эта формула, которую мы будем сокращенно обозначать через $\delta_{n}$, выглядит так:

$$
\begin{aligned}
& \left(\forall x_{1}\right)\left(\forall x_{2}\right) \cdots\left(\forall x_{n+2}\right)\left(\exists y_{1}\right)\left(\exists y_{2}\right) \cdots\left(\exists y_{n+2}\right) \\
& \quad\left[( x _ { 1 } \sqcap x _ { 2 } \sqcap \cdots \sqcap x _ { n + 2 } = \mathbf { 0 } ) \rightarrow \left(\left(x_{1} \leqslant y_{1}\right) \wedge\left(x_{2} \leqslant y_{2}\right) \wedge \cdots \wedge\left(x_{n+2} \leqslant y_{n+2}\right)\right.\right. \\
& \left.\left.\wedge\left(y_{1} \sqcap y_{2} \sqcap \cdots \sqcap y_{n+2}=\mathbf{0}\right) \wedge\left(y_{1} \sqcup y_{2} \sqcup \cdots \sqcup y_{n+2}=\mathbf{1}\right)\right)\right] .
\end{aligned}
$$

Теорема Хеммингсена утверждает, что для бикомпактньх пространств $\operatorname{dim} X \leqslant n$ в том и только том случае, если в решетке $2^{X}$ выполнена формула $\delta_{n}$.

С помощью стандартного рассуждения, связанного с ужатиями и раздутиями, можно показать, что для бикомпакта (хаусдорфова бикомпактного пространства) $X \operatorname{dim} X \leqslant n$ тогда и только тогда, когда в некоторой (любой) решеточной базе замкнутых множеств в этом пространстве выполнена формула $\delta_{n}$.

3.2. Большая индуктивная размерность. Определение большой индуктивной размерности довольно легко можно переформулировать в терминах замкнутых множеств. Перегородку $C$ между двумя непересекаюшимися замкнутыми множествами $A$ и $B$ можно определить как пару замкнутых множеств $F$ и $G$ таких, что $F \cup G=X$, $F \cap A=\varnothing$ и $G \cap B=\varnothing$ : пересечение $F \cap G$ является перегородкой между $A$ и $B$; таким образом, приведенная ниже формула, которую мы обозначаем через $\operatorname{part}(u, x, y, a)$, утверждает, что $u$ является перегородкой между $x$ и $y$ в (под)пространстве $a$ :

$$
(\exists f)(\exists g)((x \sqcap f=\mathbf{0}) \wedge(y \sqcap g=\mathbf{0}) \wedge(f \sqcup g=a) \wedge(f \sqcap g=u)) .
$$


Эта формула позволяет рекурсивно определить формулу $I_{n}(a)$ для большой индуктивной размерности:

$$
(\forall x)(\forall y)(\exists u)\left[((x \leqslant a) \wedge(y \leqslant a) \wedge(x \sqcap y=0)) \rightarrow\left(\operatorname{part}(u, x, y, a) \wedge I_{n-1}(u)\right)\right] ;
$$

основанием рекурсии служит формула $I_{-1}(a)$, согласно которой $a=\mathbf{0}$.

Таким образом, для бикомпактного пространства $X$ Ind $X \leqslant n$ тогда и только тогда, когда в $2^{X}$ вьполнена формула $I_{n}(\mathbf{1})$. Вообще, если $X$ имеет решеточную базу $\mathscr{B}$ замкнутых множеств, в которой выполнена формула $I_{n}(\mathbf{1})$, то Ind $X \leqslant n$; это легко получается по индукции - достаточно заметить, что $\{F \in \mathscr{B}: F \subseteq A\}$ является решеточной базой замкнутых множеств в подпространстве $A$, если $A \in \mathscr{B}$.

Обратное неверно: в решеточной базе замкнутых множеств в пространстве $X$, для которого Ind $X \leqslant n$, не обязательно должна выполняться формула $I_{n}(\mathbf{1})$. Простейший пример - единичньй отрезок $[0,1]$ с решеточной базой, порожденной предбазой

$$
\{[0, q]: q \text { рационально }\} \cup\{[p, 1]: p \text { иррационально }\} \text {. }
$$

В этой решетке формула $I_{n}(\mathbf{1})$ не вьполнена ни для какого $n$.

3.3. Размерность Dimensionsgrad. Согласно данному вьше определению разрез между двумя (непересекающимися) замкнутьми множествами $A$ и $B$ - это замкнутое множество $C$ такое, что любой континуум в объемлющем пространстве, пересекающийся с $A$ и $B$, пересекается и с $C$. Обозначим через $\operatorname{conn}(a)$ формулу

$$
(\forall x)(\forall y)[((x \sqcap y=\mathbf{0}) \wedge(x \sqcup y=a)) \rightarrow((x=\mathbf{0}) \vee(x=a))],
$$

которая означает " $a$ связно", а через $\operatorname{cut}(u, x, y, a)$ формулу

$$
(\forall v)[((v \leqslant a) \wedge \operatorname{conn}(v) \wedge(v \sqcap x \neq \mathbf{0}) \wedge(v \sqcap y \neq \mathbf{0})) \rightarrow(v \sqcap u \neq \mathbf{0})],
$$

которая означает " $u$ является разрезом между $x$ и $y$ в (под)пространстве $a$ "; в результате мы получим следующее рекурсивное определение формулы $\Delta_{n}(a)$ для размерности Dimensionsgrad:

$$
(\forall x)(\forall y)(\exists u)\left[((x \leqslant a) \wedge(y \leqslant a) \wedge(x \sqcap y=\mathbf{0})) \rightarrow\left(\operatorname{cut}(u, x, y, a) \wedge \Delta_{n-1}(u)\right)\right] ;
$$

как и выше, $\Delta_{-1}(a)$ обозначает " $a=0$ ".

Как и в случае большой индуктивной размерности, $\operatorname{Dg} X \leqslant n$ тогда и только тогда, когда в $2^{X}$ выполнена формула $\Delta_{n}(\mathbf{1})$. Тот же пример, что и выше, показьвает, что равенство $\operatorname{Dg} X=1$ не обязательно означает, что во всякой решеточной базе замкнутых множеств выполнена формула $\Delta_{n}(\mathbf{1})$ для какого-нибудь $n$. Возможно даже, в некоторой решеточной базе замкнутых множеств в пространстве $X$ формула $\Delta_{0}(\mathbf{1})$ верна, но $\operatorname{Dg} X>0$. Пример - единичньй отрезок и решеточная база, порожденная предбазой $\left\{[0, q] \cup\left\{q+2^{-n}: n \in \omega\right\}: q\right.$ рационально $\} \cup\left\{[p, 1] \cup\left\{p-2^{-n}: n \in \omega\right\}: p\right.$ иррационально $\}$. В этой решеточной базе формула $\Delta_{0}(\mathbf{1})$ выполнена автоматически, поскольку в ней нет нетривиальных связных элементов. 


\section{4. Элементарность}

В этом разделе мы фиксируем бикомпакт $X$ и элементарную подрешетку $L$ решетки $2^{X}$ с волмэновским представлением $w L$.

4.1. Равенство $\operatorname{dim} w L=\operatorname{dim} X$. Этот факт довольно хорошо известен, но для полноты мы наметим его доказательство. Из замечаний, сделанных в предыдушем разделе, мы знаем, что $\operatorname{dim} w L$ - наименьшее натуральное число $n$, для которого в $L$ вьполнена формула $\delta_{n}$. Следовательно, нам нужно показать, что в $L$ вьполнена формула $\delta_{n}$ тогда и только тогда, когда в $2^{X}$ вьполнена формула $\delta_{n}$. Достаточность доказьвается непосредственно: если в $2^{X}$ вьполнена формула $\delta_{n}$, то она вьполнена и в $L$ : всякая $(n+2)$-ка $\left(x_{1}, \ldots, x_{n+2}\right)$ из $L$ определяет с помощью формулы $\delta_{n}$ уравнение, которое имеет решение $\left(y_{1}, \ldots, y_{n+2}\right)$ в $2^{X}$ и, следовательно, в $L$. Необходимость доказьвается от противного: отрицание формулы $\delta_{n}$ само является уравнением с параметрами 0 и 1 и неизвестными $x_{1}, \ldots, x_{n+2}$; если бы оно имело решение в $2^{X}$, то оно имело бы решение и в $L$.

4.2. Оценка Ind $w L \leqslant$ Ind $X$. Как и вьше, в $L$ выполнена формула $I_{n}(\mathbf{1})$ тогда и только тогда, когда в $2^{X}$ вьполнена формула $I_{n}(\mathbf{1}):$ как $I_{n}(\mathbf{1})$, так и ее отрицание порождают уравнения с параметрами в $L$ и решениями в $2^{X}$, а значит, и в $L$. В п. 3.2 мы показали, что Ind $w L \leqslant n$, если в $L$ вьполнена формула $I_{n}(\mathbf{1})$; этого достаточно для выполнения неравенства Ind $w L \leqslant \operatorname{Ind} X$.

4.3. Оценка $\operatorname{Dg} w L \leqslant \operatorname{Dg} X$. Как и выше, можно показать, что в $L$ вьполнена формула $\Delta_{n}(\mathbf{1})$, если и только если в $2^{X}$ выполнена формула $\Delta_{n}(\mathbf{1})$. Однако в п. 3.3 было показано, что оценка $\operatorname{Dg} w L \leqslant n$ не вытекает автоматически из того, что в $L$ вьполнена формула $\Delta_{n}(\mathbf{1})$. Таким образом, доказательство требует дополнительных усилий. Мы докажем следуюшее утверждение индукцией по $n$.

ПРЕДЛОЖЕНИЕ 4.1. Пусть $X-$ бикомпакт, $\operatorname{Dg} X \leqslant n u L$ - әлементарная подрешетка в $2^{X}$. Тогда $\operatorname{Dg} w L \leqslant n$.

ДокАЗАтЕльство. Элемент $A$ решетки $L$ является замкнутым подмножеством $X$; с другой стороны, он определяет элемент замкнутой базы в $w L$; мы обозначаем этот элемент базы через $A_{L}$.

Пусть $P$ и $Q$ - замкнутые непересекаюшиеся множества в $w L$. Поскольку $L$ является решеточной базой замкнутых множеств в $w L$, найдутся непересекающиеся $A, B \in L$ такие, что $P \subseteq A_{L}$ и $Q \subseteq B_{L}$.

В $X$ имеется разрез cut $C$ между $A$ и $B$, для которого $\operatorname{Dg} C \leqslant n-1$; в силу элементарности мы можем считать, что $C \in L$. Действительно, очевидно, уравнение $\operatorname{cut}(x, A$, $B, \mathbf{1}) \wedge \Delta_{n-1}(x)$, параметры которого принадлежат $L$, разрешимо в $2^{X}$; значит, оно разрешимо и в $L$.

Мы должны показать, что замкнутое множество $C_{L}$, которое представлено элементом $C$ в $w L$, является разрезом между $A_{L}$ и $B_{L}\left(\right.$ а значит, и между $P$ и $Q$ ) и $\operatorname{Dg}\left(C_{L}\right) \leqslant$ $n-1$.

Второе утверждение доказьвается по индукции: $C_{L}$ является волмэновским представлением решетки $\{x \in L: x \subseteq C\}$, и эта решетка является элементарной подрешеткой решетки $\left\{x \in 2^{X}: x \subseteq C\right\}$.

Чтобы доказать первое утверждение, предположим, что в $w L$ существует замкнутое множество $K$, которое пересекает $A_{L}$ и $B_{L}$, но не пересекает $C_{L}$. Возьмем $H \in L$, для 
которого $K \subseteq H_{L}$ и $H \cap C=\varnothing$. Заметим, что $H$ несвязно, потому что оно пересекается с $A$ и с $B$, но не пересекается с $C$. Следовательно, применяя определение элементарности к формуле $\neg \operatorname{conn}(H)$, мы можем найти ненулевые непересекающиеся элементы $F$ и $G$ решетки $L$, для которых $H=F \cup G$. Множество $H_{L}$ является дизъюнктным объединением множеств $F_{L}$ и $G_{L}$; однако это не влечет несвязности $K$, поскольку вполне может оказаться, что $K \subseteq F_{L}$ или $K \subseteq G_{L}$, так что множества $F$ и $G$ нужно выбирать с большей осмотрительностью.

Воспользуемся тем, что никакая компонента множества $H$ в $X$ не пересекается одновременно с $A$ и $B$. Поскольку разложение $H$ на компоненты полунепрерывно сверху [ 1 , п. 6.2.21], мы можем найти два непересекаюшихся замкнутых множества $F$ и $G$, для которых $F \cup G=H, A \cap H \subseteq F$ и $B \cap H \subseteq G$. Существование таких $F$ и $G$ в $L$ вытекает из элементарности.

Рассуждаем в $w L$ : из включения $K \subseteq H_{L}$ вытекает, что

$$
\varnothing \neq K \cap A_{L} \subseteq H_{L} \cap A_{L} \subseteq F_{L}, \quad \varnothing \neq K \cap B_{L} \subseteq H_{L} \cap B_{L} \subseteq G_{L}
$$

Значит, $K$ несвязно, поскольку $K \subseteq H_{L}=F_{L} \cup G_{L}$ и $F_{L} \cap G_{L}=\varnothing$.

Рассуждая от противного и пользуясь этим замечанием, мы видим, что $C_{L}$ действительно является разрезом между $A_{L}$ и $B_{L}$.

4.4. Доказательство неравенства $\operatorname{dim} X \leqslant \operatorname{Dg} X$. По теореме ЛёвенгеймаСкулема в $2^{X}$ существует счетная элементарная подрешетка $M$. Волмэновское представление $w M$ этой решетки бикомпактно и метризуемо.

Согласно теореме, доказанной в [3], имеем $\operatorname{dim} w M=\operatorname{Dg} w M$. Это равенство, а также равенство $\operatorname{dim} w M=\operatorname{dim} X$ и неравенство $\operatorname{Dg} w M \leqslant \operatorname{Dg} X$, влечет $\operatorname{dim} X \leqslant \operatorname{Dg} X$.

\section{5. Заключительные замечания}

Поскольку всякая перегородка между двумя замкнутыми множествами является также и разрезом между этими множествами, неравенство $\operatorname{Dg} X \leqslant \operatorname{Ind} X$ доказывается без особого труда для всех нормальных пространств. Из неравенства Федорчука вытекает неравенство Веденисова $\operatorname{dim} X \leqslant \operatorname{Ind} X$ для бикомпактных пространств [1, п. 7.2.8]. Методом Лёвенгейма-Скулема это неравенство можно доказать и непосредственно: в обозначениях п. 4.4 имеем

$$
\operatorname{dim} X=\operatorname{dim} w M=\text { Ind } w M \leqslant \operatorname{Ind} X
$$

(эти равенства распространяются на все нормальные пространства с помощью стоунчеховской компактификации).

Как отмечено во введении, стандартное доказательство того, что $\operatorname{dim} X \leqslant \operatorname{Ind} X$, довольно просто и прямолинейно, тогда как доказательство Федорчука неравенства $\operatorname{dim} X \leqslant \operatorname{Dg} X$, изложенное в [10], длиннее и использует рассуждения от противного для построения подходящего разреза. То же отличие наблюдается и в доказательствах, предложенных выше: в обоих случаях первьй шаг состоял в выборе (счетной) решетки $M$, в которой вьполнена формула $I_{n}(\mathbf{1})$ или $\Delta_{n}(\mathbf{1})$. Вторым шагом был вьвод неравенства Ind $w M \leqslant n$ или $\mathrm{Dg} w M \leqslant n$ соответственно. В каждом случае формула сама предлагала кандидата на роль перегородки или разреза; задача состояла в том, 
чтобы показать, что этот кандидат действительно является перегородкой или разрезом в пространстве $w M$. В случае перегородки это легко: доказательство завершается с выбором замкнутых множеств $F$ и $G$. В случае разреза мы знаем только, что наше множество-кандидат пересекается со связными элементами базы $M$, пересекающими $A$ и $B$; нужно выяснить, что это верно и для всех других континуумов из $w M$. Именно в этом месте мы опять воспользовались элементарностью - для того, чтобы показать, что $M$ уже содержит достаточно связных элементов. Таким образом, судя по всему, причина видимой сложности доказательства в случае размерности Dg кроется в том, что формула $\operatorname{cut}(u, x, y, a)$ содержит универсальные кванторы.

\section{СПИСОК ЦИТИРОВАННОЙ ЛИТЕРАТУРЫ}

[1] Engelking R. General Topology. Revised and completed edition. Sigma Series in Pure Mathematics. Berlin: Heldermann Verlag, 1989.

[2] Brouwer L. E. J. Über den natürlichen Dimensionsbegriff // Journal für die reine und angewandte Mathematik. 1913. V. 142. P. 146-152.

[3] Федорчук В. В., Левин М., Шепин Е. В. О брауэровском определении размерности // УМH. 1999. T. 54. № 2(326). C. 193-194.

[4] Fedorchuk V. V., van Mill J. Dimensionsgrad for locally connected Polish spaces // Fund. Math. 2000. V. 163. P. 77-82.

[5] Wallman H. Lattices and topological spaces // Ann. of Math. 1938. V. 39. P. 112-126.

[6] Hart K. P., van Mill J., Pol R. Remarks on hereditarily indecomposable continua // Proceedings of the 15th Summer Conference on General Topology and its Applications/1st Turkish International Conference on Topology and its Applications (Oxford, OH/Istanbul, 2000). V. 25, 2002. P. 179-206.

[7] van der Steeg B. Models in Topology. Ph.D. thesis: TU Delft, 2003.

[8] Hodges W. A Shorter Model Theory. Cambridge: Cambridge Univ. Press, 1997.

[9] Hemmingsen E. Some theorems in dimension theory for normal Hausdorff spaces // Duke Math. J. 1946. V. 13. P. 495-504.

[10] Федорчук В. В. О брауэровской размерности бикомпактов // Матем. заметки. 2003. Т. 73. № 2. C. 295-304.

Technical University Delft, the Netherlands 